\title{
The impact of a Coal-Fired Power Plant Shutdown Campaign on heavy metals emissions in China
}

Sili Zhou ${ }^{\mathrm{a}}$, Wendong Wei ${ }^{\mathrm{b}}$, Long Chen ${ }^{\mathrm{c}}$, Zengkai Zhang ${ }^{\mathrm{d}}$, Zhaohui Liu ${ }^{\mathrm{a}}$, Yi Wang ${ }^{\mathrm{a}}$, Jiayue Kong ${ }^{\mathrm{e}}$, Jiashuo $\mathrm{Li}^{\mathrm{i}}{ }^{*}$

aState Key Laboratory of Coal Combustion, School of Energy and Power Engineering, Huazhong University of Science and Technology, Wuhan, 430074, P.R. China

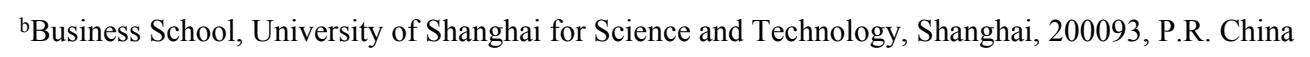

'Key Laboratory of Geographic Information Science (Ministry of Education), School of Geographic Sciences, East China Normal University, Shanghai, 200241, P.R. China

${ }^{d}$ College of Management and Economics, Tianjin University, Tianjin, 300000, P.R. China

e School of Energy and Power Engineering, Huazhong University of Science and Technology, Wuhan, 430074, P.R. China

Institute of Blue and Green Development, Shandong University, Weihai 264209, P.R. China

E-mail address: lijiashuo@sdu.edu.cn

\section{Number of pages: 35}

\section{Number of tables: 11}

Number of figures: 1

Table S1 The operating parameters of decommissioned CFPPs with accurate data

Table S2 Emission factors of decommissioned CFPPs with accurate coal consumption data

Table S3 Averaged HMs concentration in produced coal by province

Table S4 Averaged HMs concentration in consumed coal by province

Table S5 Release ratios of boilers

Table S6 Removal efficiency of APCDs for As, $\mathrm{Se}, \mathrm{Pb}, \mathrm{Cd}$ and $\mathrm{Cr}$

Table S7 Removal efficiency and speciation profiles of APCDs for $\mathrm{Hg}$

Table S8 Distribution characteristics of the APCD removal efficiencies and HMs content

Table S9 HM emissions calculated using HM content in coal consumed

Table S10 HM emissions calculated using HM content in coal locally produced

4 Table S11 Proportion of unit-specific emission reduction

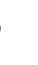

Figure S1 Parameters of decommissioned CFPPs 


\section{Coal consumption of power plants without actual data}

38

For the rest of power plants without actual data, provincial average data have been

39 applied to the calculation of coal consumption, the basic formulas can be expressed as

40 follows ${ }^{1-3}$ :

41

$$
C_{i j}=P_{i j} \times H_{i} \times E_{i}
$$

42

Where $C_{i j}$ is the estimative coal consumption of power plant $j$ in province $i, P_{i j}$

43 is the capacity of power plant $j$ in province $i$ which we couldn't get the actual operating

44 parameters; $H_{i}$ is the annual average operating hours of CFPP in province $i ; E_{i}$ is the 45 coal consumption rate of CFPP in province $i$. 
Table S1 The operating parameters of decommissioned CFPPs with accurate data

\begin{tabular}{|c|c|c|c|c|}
\hline Region & Power Plant & $\begin{array}{l}\text { Decommissioned } \\
\text { capacity (MW) }\end{array}$ & $\begin{array}{l}\text { Power generation } \\
(\mathbf{G W} \cdot \mathbf{h})\end{array}$ & $\begin{array}{l}\text { Coal consumption } \\
\text { (kt) }\end{array}$ \\
\hline \multirow{3}{*}{ Beijing } & Beijing Datang Gaojing Power Plant & 600 & 3302.77 & 1720.73 \\
\hline & Beijing Jingneng Thermal Power Co.Ltd & 800 & 5028.60 & 2210.19 \\
\hline & Guohua Beijing Thermal Power Branch (First Thermal) & 400 & 2417.02 & 778.03 \\
\hline \multirow{4}{*}{ Tianjin } & Tianjin First Thermal Power Plant & 200 & 1103.18 & 612.02 \\
\hline & Tianjin Chentang Thermal Power Co.Ltd & 135 & 413.09 & 197.14 \\
\hline & Tianjin Yongli Power Union & 120 & 261.88 & 166.16 \\
\hline & Tianjin Chentang Thermal Power Phase III & 600 & 3541.01 & 1795.28 \\
\hline \multirow{9}{*}{ Hebei } & Qinhuangdao Electric Power Company Beishan Huashi Power Plant & 24 & 23.41 & 14.00 \\
\hline & Shijiazhuang Economic Development Zone Coal and Electricity Company & 27 & 110.92 & 54.96 \\
\hline & Tangshan Xinfeng Power Generation Co.Ltd & 45 & 236.34 & 114.85 \\
\hline & Tangshan New District Thermal Power Plant & 100 & 919.61 & 685.33 \\
\hline & Hengshui Xinyi Thermal Power Company & 24 & 75.52 & 41.95 \\
\hline & Cylinder thermal power plant & 27 & 88.04 & 98.29 \\
\hline & Luohe Power Plant & 200 & 1025.82 & 786.36 \\
\hline & Zunhua Xinli Energy Development Co.Ltd & 100 & 150.71 & 99.01 \\
\hline & Hebei Huadian Shijiazhuang Luhua Thermal Power Co.Ltd & 125 & 638.16 & 268.33 \\
\hline
\end{tabular}




\begin{tabular}{|c|c|c|c|c|}
\hline & Hebei Huadian Shijiazhuang Thermal Power Co.Ltd & 25 & 132.66 & 57.08 \\
\hline & Xiandewang Coal gangue Power Plant & 12 & 37.06 & 37.05 \\
\hline & Tangshan Saide Thermal Power Company & 100 & 588.03 & 443.04 \\
\hline & Tangshan Huarun Thermal Power Co.Ltd & 200 & 1255.28 & 674.46 \\
\hline & Hebei Xingtai Power Generation Co.Ltd & 440 & 2507.80 & 1314.00 \\
\hline \multirow{14}{*}{ Shanxi } & Taiyi Power Generation Co., Ltd. & 25 & 123.72 & 56.29 \\
\hline & Shanxi Jinneng Group Datong Thermal Power Co., Ltd. & 100 & 393.87 & 271.67 \\
\hline & Jingle Power Plant & 12 & 10.34 & 15.91 \\
\hline & Gangyu Dongshan Thermal Power Plant & 6 & 41.97 & 17.49 \\
\hline & Hexi Power Plant & 36 & 71.42 & 31.32 \\
\hline & Luan Ronghai Power Plant & 12 & 64.60 & 39.35 \\
\hline & Pianguan Hongyuan Power Plant & 6 & 6.78 & 0.49 \\
\hline & Shuozhou Fuhua Electric Power Plant & 36 & 152.64 & 152.65 \\
\hline & Jingle Power Plant & 12 & 10.34 & 15.91 \\
\hline & Shentou Second Power Plant & 400 & 2506.30 & 1724.68 \\
\hline & Taixing Power Co., Ltd. & 6 & 40.94 & 39.64 \\
\hline & Xuangang (self-supplied) power plant & 12 & 22.87 & 41.90 \\
\hline & Shentou Frist Power Plant & 400 & 2551.45 & 1666.08 \\
\hline & Yongji Thermal Power Plant & 100 & 218.75 & 147.70 \\
\hline
\end{tabular}




\begin{tabular}{|c|c|c|c|c|}
\hline & Taiyuan Jinyang Power Generation Company & 5 & 27.06 & 152.05 \\
\hline & Gangyu Dongshan Thermal Power Plant & 6 & 41.97 & 69.95 \\
\hline & Qingxu Meijin Self-supplied power plant & 12 & 83.34 & 23.75 \\
\hline \multirow{11}{*}{$\begin{array}{l}\text { Inner } \\
\text { Mongolia }\end{array}$} & Hohhot Thermal Power Plant (Northern Company) & 100 & 510.36 & 279.40 \\
\hline & Yuanbaoshan Power Generation Co., Ltd. & 300 & 127.42 & 976.27 \\
\hline & Shendong Power Company Shangwan Thermal Power Plant & 24 & 92.84 & 49.53 \\
\hline & Fengzhen Power Plant (Northern Company) & 400 & 2351.28 & 1394.38 \\
\hline & Baotou Frist Power Plant (Northern Company) & 100 & 456.13 & 271.60 \\
\hline & Shenhua Zhungeer Power Generation Company & 100 & 58.03 & 331.50 \\
\hline & Damo Power Plant & 50 & 369.16 & 359.07 \\
\hline & Mengxi Cement Self-supplied Power Plant & 18 & 10.10 & 46.96 \\
\hline & Huolinhe Hongjun Aluminum Electric Company & 100 & 61.60 & 550.27 \\
\hline & Ordos Thermal Power Plant & 15 & 76.20 & 122.53 \\
\hline & Baori Hiller Power Plant & 9 & 65.23 & 88.99 \\
\hline \multirow{5}{*}{ Liaoning } & Jinzhou Thermal Power Company & 54 & 9.70 & 11.27 \\
\hline & Xiongyue Printing and Dyeing Factory Power Station & 9 & 11.30 & 4.00 \\
\hline & Huarun Power (Jinzhou) Co., Ltd. & 200 & 1364.36 & 1161.14 \\
\hline & Liaoning Chuncheng Yinzhu Thermal Power Co., Ltd. & 12 & 34.86 & 63.70 \\
\hline & Yingkou Paper Mill Power Station & 12 & 8.12 & 5.52 \\
\hline
\end{tabular}




\begin{tabular}{|c|c|c|c|c|}
\hline & State Power Investment Dalian Ganjingzi Thermal Power Plant & 100 & 27.26 & 5.64 \\
\hline & Guodian Power Dalian Development Zone Thermal Power Plant & 144 & 83.99 & 434.44 \\
\hline & Liaoyang Thermal Power Co., Ltd. & 36 & 46.50 & 29.50 \\
\hline & Liaoyang First Thermal Power Co., Ltd. & 30 & 115.24 & 105.05 \\
\hline & Tieling Thermal Power Co., Ltd. & 9 & 1.52 & 2.21 \\
\hline & Jinzhou Donggang Electric Power Co., Ltd. & 600 & 4093.07 & 3483.41 \\
\hline \multirow{5}{*}{ Jilin } & Changchun First Thermal Power Co., Ltd. & 150 & 983.75 & 623.12 \\
\hline & Siping Thermal Power Co., Ltd. & 12 & 62.12 & 46.30 \\
\hline & Hunjiang Power Plant & 200 & 892.47 & 593.48 \\
\hline & Longjing Thermal Power Plant & 15 & 50.60 & 28.75 \\
\hline & Baicheng Power Generation Company & 30 & 23.70 & 11.78 \\
\hline \multirow{8}{*}{ Jiangsu } & Huaneng Huaiyin Power Plant & 220 & 1149.75 & 517.30 \\
\hline & Sheyanggang Power Plant & 137.5 & 708.87 & 321.39 \\
\hline & Yancheng Power Plant Unit 8 & 125 & 20.96 & 10.22 \\
\hline & Yucheng Power Plant & 165 & 714.43 & 445.08 \\
\hline & Nanjing Huarun Thermal Power Co., Ltd. & 270 & 1377.42 & 661.19 \\
\hline & Suzhou Dongwu Thermal Power Co., Ltd. & 6 & 46.20 & 27.65 \\
\hline & Nanhua Thermal Power Plant & 30 & 55.67 & 25.42 \\
\hline & Huadian Yangzhou Power Plant & 220 & 935.97 & 430.27 \\
\hline
\end{tabular}




\begin{tabular}{|c|c|c|c|}
\hline Xuzhou Power Co., Ltd. & 440 & 2430.52 & 1208.23 \\
\hline Jiangyin Xingcheng Special Steel Co., Ltd. (220kV) & 12 & 82.42 & 17.26 \\
\hline Wuxi Shuanghejian Thermal Power Plant & 24 & 75.98 & 32.29 \\
\hline Wuxi Xielian Thermal Power Plant & 112 & 681.49 & 271.50 \\
\hline Yixing Thermal Power Plant & 24 & 36.41 & 11.27 \\
\hline Jiangyin Huamei Thermal Power Plant (Huaguang) & 6 & 18.56 & 5.01 \\
\hline Suzhou Qiandeng Thermal Power Plant & 6 & 7.55 & 2.99 \\
\hline Changshu Suyu Thermal Power Plant & 12 & 74.96 & 36.71 \\
\hline Yancheng Thermal Power Plant & 30 & 137.68 & 81.01 \\
\hline Wuxi Xinyang Thermal Power Company Thermal Power Plant & 6 & 14.29 & 8.92 \\
\hline Jiangsu Tianhong Chemical Co., Ltd. (Xinyuan) & 24 & 13.96 & 9.56 \\
\hline Jiangyin Sanfangxiang Thermal Power Plant & 33 & 104.50 & 47.91 \\
\hline Changzhou First Thermal Power Plant & 18 & 54.90 & 25.18 \\
\hline Zhenjiang Dagang Thermal Power Plant & 12 & 37.78 & 13.81 \\
\hline Hongze Thermal Power Plant & 21 & 21.27 & 12.76 \\
\hline Liyang Thermal Power Plant & 12 & 40.53 & 33.38 \\
\hline Jiangyin Chengxing Phosphorus Thermal Power Plant & 24 & 93.78 & 42.55 \\
\hline Zhangjiagang Aoyang Co., Ltd. Thermal Power Plant & 12 & 36.40 & 7.48 \\
\hline Jiangyan Fertilizer Plant Thermal Power Station & 3 & 9.81 & 4.31 \\
\hline
\end{tabular}




\begin{tabular}{|c|c|c|c|}
\hline Changzhou Zhenhua Cogeneration Co., Ltd. & 30 & 68.16 & 39.52 \\
\hline Danyang Fertilizer Factory & 12 & 21.70 & 6.81 \\
\hline Guodian Jianbi Power Plant & 330 & 1871.45 & 902.20 \\
\hline Xuzhou Huamei Power Plant & 55 & 355.57 & 458.27 \\
\hline Zhangjiagang Hengdong Thermal Power Co., Ltd. & 50 & 358.80 & 190.18 \\
\hline Nanjing Second Thermal Power Plant & 235 & 1426.73 & 776.59 \\
\hline Taicang Port Environmental Protection Power Co., Ltd. & 270 & 1649.29 & 785.78 \\
\hline Suzhou Thermal Power Plant & 12 & 26.34 & 8.45 \\
\hline Wujiang Thermal Power Plant & 11.5 & 25.67 & 11.14 \\
\hline Qidong Blue Sky Thermal Power Co., Ltd. & 12 & 28.84 & 19.31 \\
\hline Jiangyin Huaxi Thermal Power Plant & 24 & 126.49 & 68.46 \\
\hline Jiangyin Huaqiang Stainless Steel Material Factory & 12 & 28.02 & 4.74 \\
\hline Wujiang Yingxiang Thermal Power Plant & 6 & 2.96 & 1.31 \\
\hline Nantong Acetate Fiber Co. & 1 & 5.02 & 0.95 \\
\hline Xinyi Tongxin Thermal Power Plant & 30 & 119.00 & 88.99 \\
\hline Xuzhou West District Environmental Protection Thermal Power Co., Ltd. & 30 & 170.00 & 119.56 \\
\hline Wujin Hutang Thermal Power Plant & 12 & 49.94 & 22.61 \\
\hline Jiangyin Hailan Group Thermal Power Plant & 12 & 91.07 & 41.45 \\
\hline Zhangjiagang bonded Zone Thermal Power Plant & 24 & 81.13 & 28.88 \\
\hline
\end{tabular}




\begin{tabular}{|c|c|c|c|c|}
\hline & Xuzhou Huamei Power Plant & 55 & 355.57 & 229.13 \\
\hline & Yixing Xielian Thermal Power Plant & 84 & 573.02 & 283.95 \\
\hline & Yancheng Power Plant Unit 9 & 135 & 614.58 & 280.77 \\
\hline & Jiangyin Xingcheng Special Steel Co., Ltd. (220kV) & 12 & 82.42 & 17.26 \\
\hline & Wuxian Waikuatang Thermal Power Plant & 6 & 6.09 & 3.81 \\
\hline & Wujiang Thermal Power Plant & 11.5 & 25.67 & 12.59 \\
\hline & Taicang Xinzhonggang Xiexin Thermal Power Plant & 45 & 241.37 & 170.13 \\
\hline & Yancheng Thermal Power Plant & 30 & 137.68 & 81.01 \\
\hline & Xuzhou Jinshanqiao Thermal Power Plant & 15 & 109.00 & 41.70 \\
\hline & Jintai Environmental Protection Thermal Power Plant & 18 & 157.20 & 77.69 \\
\hline & Nanjing Xinsu Power Plant & 12 & 15.90 & 13.38 \\
\hline & Jiangsu Yangguan Xinqiao Thermal Power Co., Ltd. & 12 & 41.67 & 11.52 \\
\hline & Zhangjiagang Jinliu Thermal Power Plant & 15 & 50.82 & 17.99 \\
\hline & Wujiang Linhu Thermal Power Co., Ltd. & 24 & 91.53 & 55.41 \\
\hline & Xuzhou Bashan Environmental Protection Thermal Power Plant & 30 & 191.40 & 138.66 \\
\hline & Suzhou Iron and Steel Plant & 6.75 & 35.80 & 18.31 \\
\hline & Shaoxing Thermal Power Co., Ltd. & 27 & 265.11 & 17.64 \\
\hline Zhejiang & Henghua Thermal Power Plant & 24 & 117.09 & 56.59 \\
\hline & Pinghu Thermal Power Plant & 12 & 41.09 & 23.25 \\
\hline
\end{tabular}




\begin{tabular}{|c|c|c|c|c|}
\hline & Ningbo Zhenhai Thermal Power Plant Co., Ltd. & 54 & 36.16 & 16.15 \\
\hline & Zhejiang Zheneng Qianqing Power Generation Co., Ltd. & 125 & 68.25 & 347.82 \\
\hline & Zhejiang Zheneng Qianqing Power Generation Co., Ltd. & 125 & 68.25 & 375.64 \\
\hline & Hongshan Thermal Power Company & 18 & 102.85 & 41.42 \\
\hline & Hongbao Thermal Power Plant & 18 & 117.45 & 54.10 \\
\hline & Deqing Zhongneng Thermal Power Co., Ltd. & 9 & 33.22 & 11.24 \\
\hline & Ningbo Shunlong Electric Industry Co., Ltd. & 27 & 49.51 & 30.71 \\
\hline & Cixi Jinlun Energy Development Co., Ltd. & 24 & 0.62 & 33.01 \\
\hline & Huangyan Thermal Power Co., Ltd. & 36 & 0.00 & 31.20 \\
\hline & Hangzhou Huadian Banshan Power Generation Co., Ltd. (Coal Power) & 135 & 72.28 & 414.65 \\
\hline & Juhua Thermal Power Plant & 50 & 30.88 & 196.29 \\
\hline & Jinjiang Thermal Power Plant & 62 & 292.32 & 125.20 \\
\hline & Huashe Thermal Power Co., Ltd. & 30 & 80.11 & 38.07 \\
\hline & Xiaoshan Power Plant & 260 & 141.85 & 664.49 \\
\hline & Zhejiang Xinfeng Thermal Power Co., Ltd. & 49 & 90.82 & 31.98 \\
\hline & Zaozhuang Nanjiao Thermal Power Co., Ltd. & 66 & 125.71 & 88.97 \\
\hline Shondono & Lubei Enterprise Group Thermal Power Plant & 250 & 620.18 & 301.41 \\
\hline & Langgou Thermal Power Plant & 12 & 15.97 & 9.28 \\
\hline & Guozhuang Power Plant & 6 & 7.31 & 4.36 \\
\hline
\end{tabular}




\begin{tabular}{|c|c|c|c|}
\hline Taoyang Power Plant & 6.5 & 7.63 & 7.01 \\
\hline Yangzhuang Power Plant & 15 & 13.77 & 14.48 \\
\hline Chazhuang Power Plant & 13 & 54.96 & 29.48 \\
\hline Caozhuang Power Plant & 30 & 22.20 & 30.55 \\
\hline Chazhuang Low Calorific Value Power Plant & 60 & 136.28 & 57.92 \\
\hline Baizhuang low calorific value power plant & 60 & 203.52 & 102.20 \\
\hline Sun Paper Thermal Power Plant \#1 Machine & 30 & 185.01 & 102.57 \\
\hline Centennial power & 110 & 696.18 & 379.73 \\
\hline Wusuotun Shengjian Power Plant & 66 & 296.56 & 266.15 \\
\hline Yanggu Xiangguang Thermal Power Co., Ltd. & 60 & 184.58 & 91.92 \\
\hline Xuchang Mine Power Plant & 12 & 57.77 & 39.44 \\
\hline Yanggu Thermal Power Plant & 60 & 212.10 & 141.64 \\
\hline Yinhe Thermal Power Plant & 30 & 168.04 & 120.29 \\
\hline Dongming Petrochemical Thermal Power Co., Ltd. & 6 & 17.82 & 8.75 \\
\hline Yaxing Chemical Industry Co., Ltd. & 6 & 0.00 & 17.75 \\
\hline Zibo Hongda Thermal Power Co., Ltd. & 36 & 0.00 & 120.82 \\
\hline Yantai Power Plant & 110 & 603.88 & 298.11 \\
\hline Linyi Power Plant & 140 & 807.85 & 375.17 \\
\hline Binzhou Chemical Group Thermal Power Plant & 30 & 231.11 & 10.87 \\
\hline
\end{tabular}




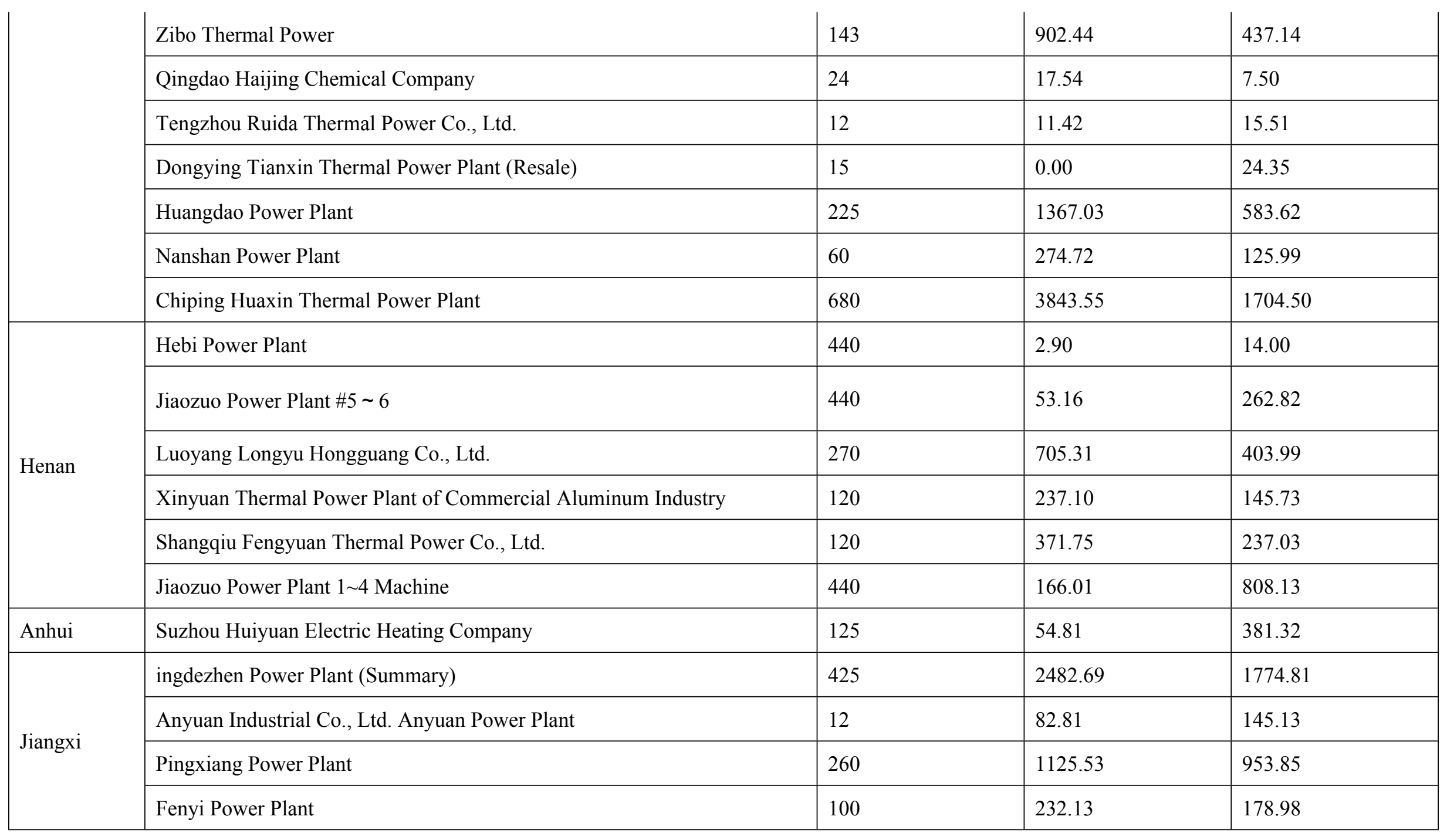




\begin{tabular}{|c|c|c|c|c|}
\hline & Jiujiang Power Plant & 840 & 4485.60 & 1129.97 \\
\hline \multirow{2}{*}{ Hubei } & Jingmen Power Plant & 440 & 1581.52 & 868.75 \\
\hline & Changyuan First Power Generation Co., Ltd. & 220 & 1061.16 & 513.65 \\
\hline \multirow{5}{*}{ Hunan } & Datang Xiangtan Power Plant & 79 & 297.77 & 151.56 \\
\hline & Tang Leiyang Power Plant & 36 & 179.03 & 85.78 \\
\hline & Baisha Power Company Baisha Plant & 24 & 106.33 & 34.61 \\
\hline & Yiyang Stone Coal Power Plant & 12 & 46.89 & 290.05 \\
\hline & Hunan Zhicheng Chemical Thermal Power Plant & 18 & 18.06 & 6.78 \\
\hline \multirow{5}{*}{ Guangdong } & Guangzhou Paper Factory & 100 & 447.86 & 278.07 \\
\hline & Guangzhou Meishan Sugar Factory & 12 & 58.56 & 33.65 \\
\hline & Doumen County Baijiao Sugar Factory (Yili) & 27 & 57.60 & 78.84 \\
\hline & Lianzhou Power Plant $1 \sim 4$ & 520 & 2841.53 & 1428.88 \\
\hline & Shantou First Thermal Power Plant & 30 & 53.44 & 36.71 \\
\hline \multirow{3}{*}{ Fujian } & Deyi Thermal Power Plant & 24 & 74.11 & 47.31 \\
\hline & Yongding Jinye Thermal Power Co., Ltd. & 50 & 129.52 & 77.65 \\
\hline & Shaowu Power Plant (expansion) & 250 & 235.39 & 184.92 \\
\hline \multirow{3}{*}{ Sichuan } & Luzhou Huangpu Electric Power Co., Ltd. & 30 & 25.45 & 29.27 \\
\hline & Guodian Minjiang Power Generation Co., Ltd. & 270 & 1377.06 & 1185.06 \\
\hline & Weiyuan Nengxing Power Generation Company & 12 & 27.31 & 61.71 \\
\hline
\end{tabular}




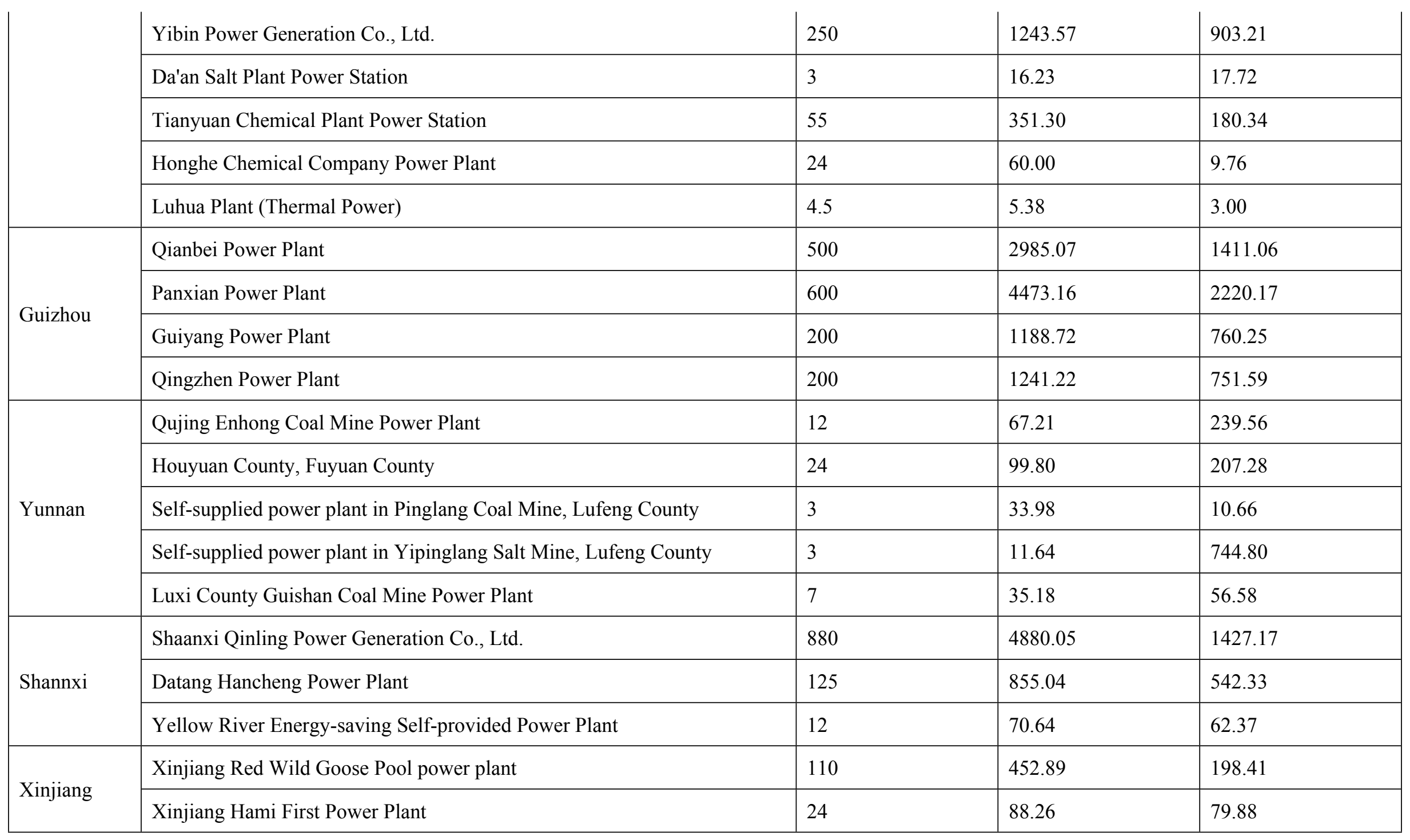




\begin{tabular}{|l|l|l|l|}
\hline Wusu Thermal Power & 15 & 84.98 & 80.57 \\
\cline { 2 - 4 } Yili First Thermal Power Plant & 54.8 & 260.33 & 214.29 \\
\hline Tashidian Thermal Power Plant & 99 & 744.41 & 501.10 \\
\hline Beitun Thermal Power Plant & 12 & 72.16 & 69.00 \\
\hline Lasqui Thermal Power Plant & 24 & 46.82 & 69.00 \\
\hline Xinjiang Weihuliang Power Generation Co., Ltd. & 250 & 1356.65 & 605.04 \\
\hline Kashgar Power Generation Company & 100 & 410.42 & 324.61 \\
\hline Xinjiang Tiechanggou Power Generation Co., Ltd. & 74 & 738.73 & 326.20 \\
\hline Baicheng Power Plant & 99 & 372.01 & 512.90 \\
\hline Yili Second Thermal Power Plant & 50 & 248.34 \\
\hline
\end{tabular}


Table S2 Emission factors of decommissioned CFPPs with accurate coal consumption data ${ }^{4}$

\begin{tabular}{|c|c|c|c|c|c|c|c|}
\hline \multirow{2}{*}{ Region } & \multirow{2}{*}{ Power Plant } & \multicolumn{6}{|c|}{ Emission Factor (g/t) } \\
\hline & & Hg & As & Se & $\mathbf{P b}$ & Cd & $\mathbf{C r}$ \\
\hline \multirow{3}{*}{ Beijing } & Beijing Datang Gaojing Power Plant & 0.045 & 0.104 & 0.227 & 0.270 & 0.005 & 0.123 \\
\hline & Beijing Jingneng Thermal Power Plant & 0.045 & 0.104 & 0.227 & 0.270 & 0.005 & 0.123 \\
\hline & Guohua Beijing Thermal Power Branch & 0.045 & 0.104 & 0.227 & 0.270 & 0.005 & 0.123 \\
\hline \multirow{4}{*}{ Tianjin } & Tianjin First Thermal Power Plant & 0.115 & 0.491 & 0.944 & 1.175 & 0.028 & 0.853 \\
\hline & Tianjin Chentang Thermal Power Co.Ltd & 0.115 & 0.491 & 0.944 & 1.175 & 0.028 & 0.853 \\
\hline & Tianjin Yongli Power Union & 0.115 & 0.491 & 0.944 & 1.175 & 0.028 & 0.853 \\
\hline & Tianjin Chentang Thermal Power Phase III & 0.115 & 0.491 & 0.944 & 1.175 & 0.028 & 0.853 \\
\hline \multirow{8}{*}{ Hebei } & Qinhuangdao Electric Power Company Beishan Huashi Power Plant & 0.104 & 0.633 & 0.498 & 1.210 & 0.010 & 0.782 \\
\hline & Shijiazhuang Economic Development Zone Coal and Electricity Company & 0.104 & 0.633 & 0.498 & 1.210 & 0.010 & 0.782 \\
\hline & Tangshan Xinfeng Power Generation Co.Ltd & 0.104 & 0.633 & 0.498 & 1.210 & 0.010 & 0.782 \\
\hline & Tangshan New District Thermal Power Plant & 0.104 & 0.633 & 0.498 & 1.210 & 0.010 & 0.782 \\
\hline & Hengshui Xinyi Thermal Power Company & 0.108 & 0.677 & 0.486 & 1.282 & 0.011 & 0.777 \\
\hline & Cylinder thermal power plant & 0.108 & 0.677 & 0.486 & 1.282 & 0.011 & 0.777 \\
\hline & Luohe Power Plant & 0.104 & 0.633 & 0.498 & 1.210 & 0.010 & 0.782 \\
\hline & Zunhua Xinli Energy Development Co.Ltd & 0.108 & 0.677 & 0.486 & 1.282 & 0.011 & 0.777 \\
\hline
\end{tabular}




\begin{tabular}{|c|c|c|c|c|c|c|c|}
\hline & Hebei Huadian Shijiazhuang Luhua Thermal Power Co.Ltd & 0.108 & 0.677 & 0.486 & 1.282 & 0.011 & 0.777 \\
\hline & Hebei Huadian Shijiazhuang Thermal Power Co.Ltd & 0.108 & 0.677 & 0.486 & 1.282 & 0.011 & 0.777 \\
\hline & Xiandewang Coal gangue Power Plant & 0.104 & 0.633 & 0.498 & 1.210 & 0.010 & 0.782 \\
\hline & Tangshan Saide Thermal Power Company & 0.104 & 0.633 & 0.498 & 1.210 & 0.010 & 0.782 \\
\hline & Tangshan Huarun Thermal Power Co.Ltd & 0.104 & 0.633 & 0.498 & 1.210 & 0.010 & 0.782 \\
\hline & Hebei Xingtai Power Generation Co.Ltd & 0.108 & 0.677 & 0.486 & 1.282 & 0.011 & 0.777 \\
\hline \multirow{13}{*}{ Shanxi } & Taiyi Power Generation Co., Ltd. & 0.059 & 0.094 & 0.223 & 0.246 & 0.006 & 0.106 \\
\hline & Shanxi Jinneng Group Datong Thermal Power Plant & 0.097 & 0.448 & 0.910 & 1.075 & 0.028 & 0.761 \\
\hline & Jingle Power Plant & 0.097 & 0.448 & 0.910 & 1.075 & 0.028 & 0.761 \\
\hline & Gangyu Dongshan Thermal Power Plant & 0.049 & 0.035 & 1.187 & 0.228 & 0.016 & 0.824 \\
\hline & Hexi Power Plant & 0.100 & 0.479 & 0.889 & 1.138 & 0.029 & 0.757 \\
\hline & Luan Ronghai Power Plant & 0.049 & 0.035 & 1.187 & 0.228 & 0.016 & 0.824 \\
\hline & Pianguan Hongyuan Power Plant & 0.097 & 0.448 & 0.910 & 1.075 & 0.028 & 0.761 \\
\hline & Shuozhou Fuhua Electric Power Plant & 0.097 & 0.448 & 0.910 & 1.075 & 0.028 & 0.761 \\
\hline & Jingle Power Plant & 0.097 & 0.448 & 0.910 & 1.075 & 0.028 & 0.761 \\
\hline & Shentou Second Power Plant & 0.097 & 0.448 & 0.910 & 1.075 & 0.028 & 0.761 \\
\hline & Taixing Power Co., Ltd. & 0.097 & 0.448 & 0.910 & 1.075 & 0.028 & 0.761 \\
\hline & Captive Power Plant of Xuangang & 0.097 & 0.448 & 0.910 & 1.075 & 0.028 & 0.761 \\
\hline & Shentou Frist Power Plant & 0.097 & 0.448 & 0.910 & 1.075 & 0.028 & 0.761 \\
\hline
\end{tabular}




\begin{tabular}{|c|c|c|c|c|c|c|c|}
\hline & Yongji Thermal Power Plant & 0.097 & 0.448 & 0.910 & 1.075 & 0.028 & 0.761 \\
\hline & Taiyuan Jinyang Power Generation Company & 0.097 & 0.448 & 0.910 & 1.075 & 0.028 & 0.761 \\
\hline & Gangyu Dongshan Thermal Power Plant & 0.100 & 0.479 & 0.889 & 1.138 & 0.029 & 0.757 \\
\hline & Captive power plant of Qingxu Meijin & 0.097 & 0.448 & 0.910 & 1.075 & 0.028 & 0.761 \\
\hline \multirow{11}{*}{$\begin{array}{l}\text { Inner } \\
\text { Mongolia }\end{array}$} & Hohhot Thermal Power Plant (Northern Company) & 0.114 & 0.732 & 0.285 & 1.196 & 0.005 & 0.489 \\
\hline & Yuanbaoshan Power Generation Power Plant & 0.110 & 0.685 & 0.291 & 1.129 & 0.005 & 0.492 \\
\hline & Shendong Power Company Shangwan Thermal Power Plant & 0.110 & 0.685 & 0.291 & 1.129 & 0.005 & 0.492 \\
\hline & Fengzhen Power Plant (Northern Company) & 0.114 & 0.732 & 0.285 & 1.196 & 0.005 & 0.489 \\
\hline & Baotou Frist Power Plant (Northern Company) & 0.110 & 0.685 & 0.291 & 1.129 & 0.005 & 0.492 \\
\hline & Shenhua Zhungeer Power Generation Company & 0.110 & 0.685 & 0.291 & 1.129 & 0.005 & 0.492 \\
\hline & Damo Power Plant & 0.067 & 0.144 & 0.071 & 0.258 & 0.001 & 0.068 \\
\hline & Captive Power Plant of Mengxi Cement & 0.110 & 0.685 & 0.291 & 1.129 & 0.005 & 0.492 \\
\hline & Huolinhe Hongjun Aluminum Electric Company & 0.067 & 0.144 & 0.071 & 0.258 & 0.001 & 0.068 \\
\hline & Ordos Thermal Power Plant & 0.067 & 0.144 & 0.071 & 0.258 & 0.001 & 0.068 \\
\hline & Baori Hiller Power Plant & 0.110 & 0.685 & 0.291 & 1.129 & 0.005 & 0.492 \\
\hline \multirow{4}{*}{ Liaoning } & Jinzhou Thermal Power Company & 0.089 & 0.633 & 0.289 & 0.937 & 0.006 & 0.727 \\
\hline & Xiongyue Printing and Dyeing Factory Power Plant & 0.089 & 0.633 & 0.289 & 0.937 & 0.006 & 0.727 \\
\hline & Jinzhou Power Plant of Huarun & 0.054 & 0.133 & 0.071 & 0.214 & 0.001 & 0.101 \\
\hline & Liaoning Chuncheng Yinzhu Thermal Power Plant & 0.089 & 0.633 & 0.289 & 0.937 & 0.006 & 0.727 \\
\hline
\end{tabular}




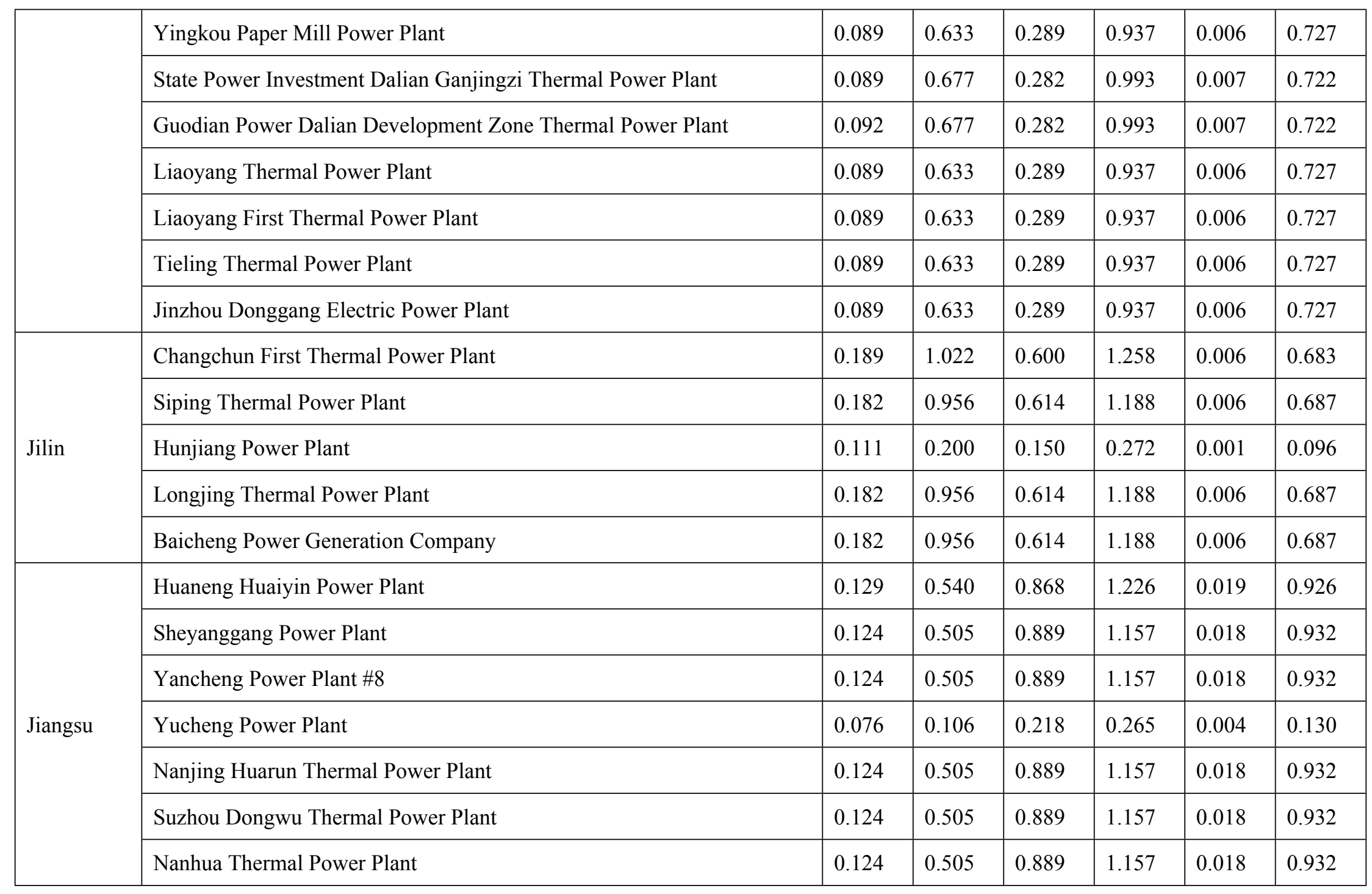




\begin{tabular}{|c|c|c|c|c|c|c|}
\hline Huadian Yangzhou Power Plant & 0.124 & 0.540 & 0.868 & 1.226 & 0.019 & 0.926 \\
\hline Xuzhou Power Power Plant & 0.124 & 0.505 & 0.889 & 1.157 & 0.018 & 0.932 \\
\hline Jiangyin Xingcheng Special Steel Power Plant & 0.129 & 0.540 & 0.868 & 1.226 & 0.019 & 0.926 \\
\hline Wuxi Shuanghejian Thermal Power Plant & 0.124 & 0.505 & 0.889 & 1.157 & 0.018 & 0.932 \\
\hline Wuxi Xielian Thermal Power Plant & 0.124 & 0.505 & 0.889 & 1.157 & 0.018 & 0.932 \\
\hline Yixing Thermal Power Plant & 0.124 & 0.505 & 0.889 & 1.157 & 0.018 & 0.932 \\
\hline Jiangyin Huamei Thermal Power Plant & 0.076 & 0.106 & 0.218 & 0.265 & 0.004 & 0.130 \\
\hline Suzhou Qiandeng Thermal Power Plant & 0.124 & 0.505 & 0.889 & 1.157 & 0.018 & 0.932 \\
\hline Changshu Suyu Thermal Power Plant & 0.076 & 0.106 & 0.218 & 0.265 & 0.004 & 0.130 \\
\hline Yancheng Thermal Power Plant & 0.124 & 0.505 & 0.889 & 1.157 & 0.018 & 0.932 \\
\hline Wuxi Xinyang Thermal Power Company Thermal Power Plant & 0.124 & 0.505 & 0.889 & 1.157 & 0.018 & 0.932 \\
\hline Jiangsu Tianhong Chemical Power Plant & 0.076 & 0.106 & 0.218 & 0.265 & 0.004 & 0.130 \\
\hline Jiangyin Sanfangxiang Thermal Power Plant & 0.076 & 0.106 & 0.218 & 0.265 & 0.004 & 0.130 \\
\hline Changzhou First Thermal Power Plant & 0.076 & 0.106 & 0.218 & 0.265 & 0.004 & 0.130 \\
\hline Zhenjiang Dagang Thermal Power Plant & 0.129 & 0.540 & 0.868 & 1.226 & 0.019 & 0.926 \\
\hline Hongze Thermal Power Plant & 0.129 & 0.540 & 0.868 & 1.226 & 0.019 & 0.926 \\
\hline Liyang Thermal Power Plant & 0.124 & 0.505 & 0.889 & 1.157 & 0.018 & 0.932 \\
\hline Jiangyin Chengxing Phosphorus Thermal Power Plant & 0.076 & 0.106 & 0.218 & 0.265 & 0.004 & 0.130 \\
\hline Zhangjiagang Aoyang Co., Ltd. Thermal Power Plant & 0.129 & 0.540 & 0.868 & 1.226 & 0.019 & 0.926 \\
\hline
\end{tabular}




\begin{tabular}{|c|c|c|c|c|c|c|}
\hline Jiangyan Fertilizer Plant Thermal Power Station & 0.124 & 0.505 & 0.889 & 1.157 & 0.018 & 0.932 \\
\hline Changzhou Zhenhua Cogeneration Power Plant & 0.076 & 0.106 & 0.218 & 0.265 & 0.004 & 0.130 \\
\hline Danyang Fertilizer Factory & 0.076 & 0.106 & 0.218 & 0.265 & 0.004 & 0.130 \\
\hline Guodian Jianbi Power Plant & 0.129 & 0.540 & 0.868 & 1.226 & 0.019 & 0.926 \\
\hline Xuzhou Huamei Power Plant & 0.124 & 0.505 & 0.889 & 1.157 & 0.018 & 0.932 \\
\hline Zhangjiagang Hengdong Thermal Power Plant & 0.129 & 0.540 & 0.868 & 1.226 & 0.019 & 0.926 \\
\hline Nanjing Second Thermal Power Plant & 0.124 & 0.505 & 0.889 & 1.157 & 0.018 & 0.932 \\
\hline Taicang Port Environmental Protection Power Plant & 0.124 & 0.505 & 0.889 & 1.157 & 0.018 & 0.932 \\
\hline Suzhou Thermal Power Plant & 0.124 & 0.505 & 0.889 & 1.157 & 0.018 & 0.932 \\
\hline Wujiang Thermal Power Plant & 0.124 & 0.505 & 0.889 & 1.157 & 0.018 & 0.932 \\
\hline Qidong Blue Sky Thermal Power Plant & 0.124 & 0.505 & 0.889 & 1.157 & 0.018 & 0.932 \\
\hline Jiangyin Huaxi Thermal Power Plant & 0.076 & 0.106 & 0.218 & 0.265 & 0.004 & 0.130 \\
\hline Jiangyin Huaqiang Stainless Steel Material Factory & 0.076 & 0.106 & 0.218 & 0.265 & 0.004 & 0.130 \\
\hline Wujiang Yingxiang Thermal Power Plant & 0.124 & 0.505 & 0.889 & 1.157 & 0.018 & 0.932 \\
\hline Nantong Acetate Fiber Co. & 0.124 & 0.505 & 0.889 & 1.157 & 0.018 & 0.932 \\
\hline Xinyi Tongxin Thermal Power Plant & 0.124 & 0.505 & 0.889 & 1.157 & 0.018 & 0.932 \\
\hline Xuzhou West District Environmental Protection Thermal Power Plant & 0.124 & 0.505 & 0.889 & 1.157 & 0.018 & 0.932 \\
\hline Wujin Hutang Thermal Power Plant & 0.124 & 0.505 & 0.889 & 1.157 & 0.018 & 0.932 \\
\hline Jiangyin Hailan Group Thermal Power Plant & 0.076 & 0.106 & 0.218 & 0.265 & 0.004 & 0.130 \\
\hline
\end{tabular}




\begin{tabular}{|c|c|c|c|c|c|c|c|}
\hline & Zhangjiagang bonded Zone Thermal Power Plant & 0.129 & 0.540 & 0.868 & 1.226 & 0.019 & 0.926 \\
\hline & Xuzhou Huamei Power Plant & 0.124 & 0.505 & 0.889 & 1.157 & 0.018 & 0.932 \\
\hline & Yixing Xielian Thermal Power Plant & 0.124 & 0.505 & 0.889 & 1.157 & 0.018 & 0.932 \\
\hline & Yancheng Power Plant \#9 & 0.124 & 0.505 & 0.889 & 1.157 & 0.018 & 0.932 \\
\hline & Jiangyin Xingcheng Special Steel Power Plant & 0.129 & 0.540 & 0.868 & 1.226 & 0.019 & 0.926 \\
\hline & Wuxian Waikuatang Thermal Power Plant & 0.124 & 0.505 & 0.889 & 1.157 & 0.018 & 0.932 \\
\hline & Wujiang Thermal Power Plant & 0.124 & 0.505 & 0.889 & 1.157 & 0.018 & 0.932 \\
\hline & Taicang Xinzhonggang Xiexin Thermal Power Plant & 0.124 & 0.505 & 0.889 & 1.157 & 0.018 & 0.932 \\
\hline & Yancheng Thermal Power Plant & 0.124 & 0.505 & 0.889 & 1.157 & 0.018 & 0.932 \\
\hline & Xuzhou Jinshanqiao Thermal Power Plant & 0.124 & 0.505 & 0.889 & 1.157 & 0.018 & 0.932 \\
\hline & Jintai Environmental Protection Thermal Power Plant & 0.124 & 0.505 & 0.889 & 1.157 & 0.018 & 0.932 \\
\hline & Nanjing Xinsu Power Plant & 0.124 & 0.505 & 0.889 & 1.157 & 0.018 & 0.932 \\
\hline & Jiangsu Yangguan Xinqiao Thermal Power Plant & 0.076 & 0.106 & 0.218 & 0.265 & 0.004 & 0.130 \\
\hline & Zhangjiagang Jinliu Thermal Power Plant & 0.129 & 0.540 & 0.868 & 1.226 & 0.019 & 0.926 \\
\hline & Wujiang Linhu Thermal Power Plant & 0.124 & 0.505 & 0.889 & 1.157 & 0.018 & 0.932 \\
\hline & Xuzhou Bashan Environmental Protection Thermal Power Plant & 0.124 & 0.505 & 0.889 & 1.157 & 0.018 & 0.932 \\
\hline & Suzhou Iron and Steel Plant & 0.124 & 0.505 & 0.889 & 1.157 & 0.018 & 0.932 \\
\hline \multirow{2}{*}{ Zhejiang } & Shaoxing Thermal Power Plant & 0.058 & 0.039 & 1.151 & 0.261 & 0.014 & 1.019 \\
\hline & Henghua Thermal Power Plant & 0.115 & 0.500 & 0.882 & 1.230 & 0.025 & 0.942 \\
\hline
\end{tabular}




\begin{tabular}{|c|c|c|c|c|c|c|c|}
\hline & Pinghu Thermal Power Plant & 0.058 & 0.039 & 1.151 & 0.261 & 0.014 & 1.019 \\
\hline & Ningbo Zhenhai Thermal Power Plant & 0.115 & 0.500 & 0.882 & 1.230 & 0.025 & 0.942 \\
\hline & Zhejiang Zheneng Qianqing Power Generation Co., Ltd. & 0.119 & 0.534 & 0.862 & 1.303 & 0.026 & 0.936 \\
\hline & Zhejiang Zheneng Qianqing Power Generation Co., Ltd. & 0.119 & 0.534 & 0.862 & 1.303 & 0.026 & 0.936 \\
\hline & Hongshan Thermal Power Company & 0.119 & 0.534 & 0.862 & 1.303 & 0.026 & 0.936 \\
\hline & Hongbao Thermal Power Plant & 0.119 & 0.534 & 0.862 & 1.303 & 0.026 & 0.936 \\
\hline & Deqing Zhongneng Thermal Power Co., Ltd. & 0.070 & 0.105 & 0.216 & 0.281 & 0.005 & 0.131 \\
\hline & Ningbo Shunlong Electric Industry Co., Ltd. & 0.115 & 0.500 & 0.882 & 1.230 & 0.025 & 0.942 \\
\hline & Cixi Jinlun Energy Development Co., Ltd. & 0.070 & 0.105 & 0.216 & 0.281 & 0.005 & 0.131 \\
\hline & Huangyan Thermal Power Co., Ltd. & 0.070 & 0.105 & 0.216 & 0.281 & 0.005 & 0.131 \\
\hline & Hangzhou Huadian Banshan Power Generation Co., Ltd. & 0.119 & 0.534 & 0.862 & 1.303 & 0.026 & 0.936 \\
\hline & Juhua Thermal Power Plant & 0.115 & 0.500 & 0.882 & 1.230 & 0.025 & 0.942 \\
\hline & Jinjiang Thermal Power Plant & 0.115 & 0.500 & 0.882 & 1.230 & 0.025 & 0.942 \\
\hline & Huashe Thermal Power Co., Ltd. & 0.070 & 0.105 & 0.216 & 0.281 & 0.005 & 0.131 \\
\hline & Xiaoshan Power Plant & 0.070 & 0.105 & 0.216 & 0.281 & 0.005 & 0.131 \\
\hline & Zhejiang Xinfeng Thermal Power Co., Ltd. & 0.119 & 0.534 & 0.862 & 1.303 & 0.026 & 0.936 \\
\hline \multirow{3}{*}{ Shandong } & Zaozhuang Nanjiao Thermal Power Co., Ltd. & 0.103 & 0.650 & 0.680 & 1.051 & 0.016 & 0.673 \\
\hline & Lubei Enterprise Group Thermal Power Plant & 0.099 & 0.608 & 0.696 & 0.992 & 0.015 & 0.677 \\
\hline & Langgou Thermal Power Plant & 0.099 & 0.608 & 0.696 & 0.992 & 0.015 & 0.677 \\
\hline
\end{tabular}




\begin{tabular}{|c|c|c|c|c|c|c|}
\hline Guozhuang Power Plant & 0.103 & 0.650 & 0.680 & 1.051 & 0.016 & 0.673 \\
\hline Taoyang Power Plant & 0.099 & 0.608 & 0.696 & 0.992 & 0.015 & 0.677 \\
\hline Yangzhuang Power Plant & 0.099 & 0.608 & 0.696 & 0.992 & 0.015 & 0.677 \\
\hline Chazhuang Power Plant & 0.060 & 0.127 & 0.171 & 0.227 & 0.003 & 0.094 \\
\hline Caozhuang Power Plant & 0.060 & 0.127 & 0.171 & 0.227 & 0.003 & 0.094 \\
\hline Chazhuang Low Calorific Value Power Plant & 0.060 & 0.127 & 0.171 & 0.227 & 0.003 & 0.094 \\
\hline Baizhuang low calorific value power plant & 0.099 & 0.608 & 0.696 & 0.992 & 0.015 & 0.677 \\
\hline Sun Paper Thermal Power Plant \#1 & 0.099 & 0.608 & 0.696 & 0.992 & 0.015 & 0.677 \\
\hline Centennial power & 0.099 & 0.608 & 0.696 & 0.992 & 0.015 & 0.677 \\
\hline Wusuotun Shengjian Power Plant & 0.099 & 0.608 & 0.696 & 0.992 & 0.015 & 0.677 \\
\hline Yanggu Xiangguang Thermal Power Co., Ltd. & 0.099 & 0.608 & 0.696 & 0.992 & 0.015 & 0.677 \\
\hline Xuchang Mine Power Plant & 0.099 & 0.608 & 0.696 & 0.992 & 0.015 & 0.677 \\
\hline Yanggu Thermal Power Plant & 0.099 & 0.608 & 0.696 & 0.992 & 0.015 & 0.677 \\
\hline Yinhe Thermal Power Plant & 0.099 & 0.608 & 0.696 & 0.992 & 0.015 & 0.677 \\
\hline Dongming Petrochemical Thermal Power Co., Ltd. & 0.060 & 0.127 & 0.171 & 0.227 & 0.003 & 0.094 \\
\hline Yaxing Chemical Industry Co., Ltd. & 0.099 & 0.608 & 0.696 & 0.992 & 0.015 & 0.677 \\
\hline Zibo Hongda Thermal Power Co., Ltd. & 0.103 & 0.650 & 0.680 & 1.051 & 0.016 & 0.673 \\
\hline Yantai Power Plant & 0.099 & 0.608 & 0.696 & 0.992 & 0.015 & 0.677 \\
\hline Linyi Power Plant & 0.099 & 0.608 & 0.696 & 0.992 & 0.015 & 0.677 \\
\hline
\end{tabular}




\begin{tabular}{|c|c|c|c|c|c|c|c|}
\hline & Binzhou Chemical Group Thermal Power Plant & 0.060 & 0.127 & 0.171 & 0.227 & 0.003 & 0.094 \\
\hline & Zibo Thermal Power & 0.103 & 0.650 & 0.680 & 1.051 & 0.016 & 0.673 \\
\hline & Qingdao Haijing Chemical Company & 0.099 & 0.608 & 0.696 & 0.992 & 0.015 & 0.677 \\
\hline & Tengzhou Ruida Thermal Power Co., Ltd. & 0.099 & 0.608 & 0.696 & 0.992 & 0.015 & 0.677 \\
\hline & Dongying Tianxin Thermal Power Plant & 0.060 & 0.127 & 0.171 & 0.227 & 0.003 & 0.094 \\
\hline & Huangdao Power Plant & 0.045 & 0.127 & 0.171 & 0.227 & 0.003 & 0.094 \\
\hline & Nanshan Power Plant & 0.099 & 0.608 & 0.696 & 0.992 & 0.015 & 0.677 \\
\hline & Chiping Huaxin Thermal Power Plant & 0.060 & 0.127 & 0.171 & 0.227 & 0.003 & 0.094 \\
\hline \multirow{6}{*}{ Henan } & Hebi Power Plant & 0.121 & 0.339 & 1.125 & 0.897 & 0.025 & 0.904 \\
\hline & Jiaozuo Power Plant $\# 5 \sim 6$ & 0.116 & 0.317 & 1.152 & 0.847 & 0.024 & 0.909 \\
\hline & Luoyang Longyu Hongguang Co., Ltd. & 0.116 & 0.317 & 1.152 & 0.847 & 0.024 & 0.909 \\
\hline & Xinyuan Thermal Power Plant of Commercial Aluminum Industry & 0.116 & 0.317 & 1.152 & 0.847 & 0.024 & 0.909 \\
\hline & Shangqiu Fengyuan Thermal Power Co., Ltd. & 0.116 & 0.317 & 1.152 & 0.847 & 0.024 & 0.909 \\
\hline & Jiaozuo Power Plant \#1 4 & 0.116 & 0.317 & 1.152 & 0.847 & 0.024 & 0.909 \\
\hline Anhui & Suzhou Huiyuan Electric Heating Company & 0.120 & 0.324 & 1.659 & 0.568 & 0.006 & 1.023 \\
\hline \multirow{3}{*}{ Jiangxi } & Jingdezhen Power Plant & 0.090 & 0.136 & 0.393 & 0.232 & 0.005 & 0.178 \\
\hline & Anyuan Industrial Co., Ltd. Anyuan Power Plant & 0.153 & 0.693 & 1.564 & 1.075 & 0.026 & 1.269 \\
\hline & Pingxiang Power Plant & 0.074 & 0.050 & 2.090 & 0.215 & 0.014 & 1.381 \\
\hline
\end{tabular}




\begin{tabular}{|c|c|c|c|c|c|c|c|}
\hline & Fenyi Power Plant & 0.090 & 0.136 & 0.393 & 0.232 & 0.005 & 0.178 \\
\hline & Jiujiang Power Plant \#1 2 & 0.148 & 0.648 & 1.601 & 1.015 & 0.025 & 1.277 \\
\hline & Jiujiang Power Plant $\# 3 \sim 4$ & 0.032 & 0.010 & 0.524 & 0.046 & 0.003 & 0.193 \\
\hline \multirow{2}{*}{ Hubei } & Jingmen Power Plant & 0.128 & 0.638 & 1.091 & 1.480 & 0.029 & 1.204 \\
\hline & Changyuan First Power Generation Co., Ltd. & 0.132 & 0.682 & 1.066 & 1.568 & 0.030 & 1.197 \\
\hline \multirow{5}{*}{ Hunan } & Datang Xiangtan Power Plant & 0.058 & 0.118 & 0.241 & 0.258 & 0.005 & 0.168 \\
\hline & Tang Leiyang Power Plant & 0.058 & 0.118 & 0.241 & 0.258 & 0.005 & 0.168 \\
\hline & Baisha Power Company Baisha Plant & 0.095 & 0.565 & 0.983 & 1.128 & 0.027 & 1.206 \\
\hline & Yiyang Stone Coal Power Plant & 0.095 & 0.565 & 0.983 & 1.128 & 0.027 & 1.206 \\
\hline & Hunan Zhicheng Chemical Thermal Power Plant & 0.099 & 0.604 & 0.960 & 1.195 & 0.028 & 1.198 \\
\hline \multirow{5}{*}{ Guangdong } & Guangzhou Paper Factory & 0.107 & 0.533 & 0.702 & 0.901 & 0.023 & 0.707 \\
\hline & Guangzhou Meishan Sugar Factory & 0.107 & 0.533 & 0.702 & 0.901 & 0.023 & 0.707 \\
\hline & Doumen County Baijiao Sugar Factory (Yili) & 0.063 & 0.104 & 0.176 & 0.195 & 0.004 & 0.099 \\
\hline & Lianzhou Power Plant \#1 4 & 0.103 & 0.498 & 0.719 & 0.851 & 0.022 & 0.711 \\
\hline & Shantou First Thermal Power Plant & 0.103 & 0.498 & 0.719 & 0.851 & 0.022 & 0.711 \\
\hline \multirow{3}{*}{ Fujian } & Deyi Thermal Power Plant & 0.090 & 0.116 & 0.147 & 0.154 & 0.002 & 0.098 \\
\hline & Yongding Jinye Thermal Power Co., Ltd. & 0.055 & 0.553 & 0.600 & 0.675 & 0.012 & 0.706 \\
\hline & Shaowu Power Plant (expansion) & 0.093 & 0.591 & 0.586 & 0.715 & 0.012 & 0.701 \\
\hline Sichuan & Luzhou Huangpu Electric Power Co., Ltd. & 0.110 & 0.657 & 0.827 & 1.293 & 0.071 & 1.278 \\
\hline
\end{tabular}




\begin{tabular}{|c|c|c|c|c|c|c|c|}
\hline & Guodian Minjiang Power Generation Co., Ltd. & 0.114 & 0.703 & 0.808 & 1.370 & 0.073 & 1.270 \\
\hline & Weiyuan Nengxing Power Generation Company & 0.110 & 0.657 & 0.827 & 1.293 & 0.071 & 1.278 \\
\hline & Yibin Power Generation Co., Ltd. & 0.110 & 0.657 & 0.827 & 1.293 & 0.071 & 1.278 \\
\hline & Da'an Salt Plant Power Station & 0.067 & 0.138 & 0.203 & 0.296 & 0.014 & 0.178 \\
\hline & Tianyuan Chemical Plant Power Station & 0.110 & 0.657 & 0.827 & 1.293 & 0.071 & 1.278 \\
\hline & Honghe Chemical Company Power Plant & 0.114 & 0.703 & 0.808 & 1.370 & 0.073 & 1.270 \\
\hline & Luhua Plant (Thermal Power) & 0.110 & 0.657 & 0.827 & 1.293 & 0.071 & 1.278 \\
\hline \multirow{4}{*}{ Guizhou } & Qianbei Power Plant & 0.137 & 0.824 & 0.984 & 1.056 & 0.032 & 1.088 \\
\hline & Panxian Power Plant & 0.137 & 0.824 & 0.984 & 1.056 & 0.032 & 1.088 \\
\hline & Guiyang Power Plant & 0.142 & 0.882 & 0.962 & 1.119 & 0.034 & 1.081 \\
\hline & Qingzhen Power Plant & 0.137 & 0.824 & 0.984 & 1.056 & 0.032 & 1.088 \\
\hline \multirow{5}{*}{ Yunnan } & Qujing Enhong Coal Mine Power Plant & 0.234 & 1.070 & 0.406 & 1.841 & 0.033 & 2.711 \\
\hline & Houyuan County, Fuyuan County & 0.243 & 1.145 & 0.397 & 1.951 & 0.034 & 2.694 \\
\hline & Captive power plant of Pinglang Coal Mine, Lufeng County & 0.234 & 1.070 & 0.406 & 1.841 & 0.033 & 2.711 \\
\hline & Captive power plant of Yipinglang Salt Mine, Lufeng County & 0.234 & 1.070 & 0.406 & 1.841 & 0.033 & 2.711 \\
\hline & Luxi County Guishan Coal Mine Power Plant & 0.234 & 1.070 & 0.406 & 1.841 & 0.033 & 2.711 \\
\hline \multirow{3}{*}{ Shannxi } & Shaanxi Qinling Power Generation Co., Ltd. & 0.137 & 0.474 & 0.907 & 1.468 & 0.029 & 1.165 \\
\hline & Datang Hancheng Power Plant & 0.083 & 0.099 & 0.222 & 0.336 & 0.006 & 0.162 \\
\hline & Captive Power Plant of Yellow River Energy-saving & 0.083 & 0.099 & 0.222 & 0.336 & 0.006 & 0.162 \\
\hline
\end{tabular}




\begin{tabular}{|c|c|c|c|c|c|c|c|}
\hline \multirow{12}{*}{ Xinjiang } & Xinjiang Red Wild Goose Pool power plant & 0.012 & 0.077 & 0.016 & 0.030 & 0.001 & 0.042 \\
\hline & Xinjiang Hami First Power Plant & 0.012 & 0.077 & 0.016 & 0.030 & 0.001 & 0.042 \\
\hline & Wusu Thermal Power & 0.012 & 0.077 & 0.016 & 0.030 & 0.001 & 0.042 \\
\hline & Yili First Thermal Power Plant & 0.021 & 0.395 & 0.063 & 0.137 & 0.005 & 0.298 \\
\hline & Tashidian Thermal Power Plant & 0.021 & 0.395 & 0.063 & 0.137 & 0.005 & 0.298 \\
\hline & Beitun Thermal Power Plant & 0.020 & 0.370 & 0.065 & 0.130 & 0.005 & 0.300 \\
\hline & Lasqui Thermal Power Plant & 0.020 & 0.370 & 0.065 & 0.130 & 0.005 & 0.300 \\
\hline & Xinjiang Weihuliang Power Generation power plant & 0.021 & 0.077 & 0.016 & 0.030 & 0.001 & 0.042 \\
\hline & Kashgar Power Generation Company & 0.021 & 0.077 & 0.016 & 0.030 & 0.001 & 0.042 \\
\hline & Xinjiang Tiechanggou Power Generation power plant & 0.021 & 0.395 & 0.063 & 0.137 & 0.005 & 0.298 \\
\hline & Baicheng Power Plant & 0.021 & 0.395 & 0.063 & 0.137 & 0.005 & 0.298 \\
\hline & Yili Second Thermal Power Plant & 0.021 & 0.395 & 0.063 & 0.137 & 0.005 & 0.298 \\
\hline
\end{tabular}


52 Table S3 Averaged HMs concentration in produced coal by province (unit: $\mathrm{g} / \mathrm{t}$ ). ${ }^{2,3,5}$

\begin{tabular}{|c|c|c|c|c|c|c|}
\hline Provinces & $\mathrm{Hg}$ & As & $\mathrm{Se}$ & $\mathrm{Pb}$ & $\mathrm{Cd}$ & $\mathrm{Cr}$ \\
\hline Anhui & 0.43 & 2.89 & 7.54 & 13.24 & 0.11 & 31.25 \\
\hline Beijing & - & - & - & - & - & - \\
\hline Chongqing & 0.31 & 5.66 & 3.69 & 30.44 & 1.22 & 28.44 \\
\hline Fujian & 0.07 & 9.93 & 1.22 & 25.53 & 0.31 & 30.48 \\
\hline Gansu & 0.27 & 4.14 & 0.51 & 8.35 & 0.08 & 23.7 \\
\hline $\begin{array}{c}\text { Guangdon } \\
\mathrm{g}\end{array}$ & 0.07 & 8.3 & 0.6 & 24.4 & 0.25 & 74 \\
\hline Guangxi & 0.33 & 16.94 & 5.03 & 29.94 & 0.41 & 116.41 \\
\hline Guizhou & 0.39 & 6.68 & 3.82 & 23.81 & 0.79 & 28.47 \\
\hline Hainan & - & - & - & - & - & - \\
\hline Hebei & 0.15 & 4.88 & 2.31 & 29.3 & 0.23 & 32.52 \\
\hline $\begin{array}{l}\text { Heilongjia } \\
\text { ng }\end{array}$ & 0.12 & 3.42 & 0.9 & 22.15 & 0.13 & 15.48 \\
\hline Henan & 0.2 & 2.2 & 4.86 & 16.78 & 0.54 & 24.94 \\
\hline Hubei & 0.2 & 5.3 & 8.76 & 47.39 & 0.36 & 40.52 \\
\hline Hunan & 0.12 & 10.59 & 3.72 & 26.29 & 0.64 & 37.03 \\
\hline $\begin{array}{c}\text { Inner } \\
\text { Mongolia }\end{array}$ & 0.22 & 5.77 & 1.1 & 26.67 & 0.1 & 13.02 \\
\hline Jiangsu & 0.69 & 2.74 & 6.11 & 20.98 & 0.06 & 19.82 \\
\hline Jiangxi & 0.16 & 7.41 & 8.39 & 19.33 & 0.56 & 39.75 \\
\hline Jilin & 0.4 & 11.57 & 4.06 & 29 & 0.15 & 23.09 \\
\hline Liaoning & 0.17 & 5.51 & 0.85 & 19.68 & 0.16 & 26.24 \\
\hline Ningxia & 0.22 & 3.65 & 4.27 & 14.05 & 1.1 & 10.63 \\
\hline Qinghai & 0.25 & 2.68 & 0.3 & 10.72 & 0.03 & 30.82 \\
\hline Shaanxi & 0.21 & 3.87 & 3.43 & 35.17 & 0.75 & 32.73 \\
\hline Shandong & 0.18 & 5.23 & 3.66 & 16.64 & 0.39 & 20.62 \\
\hline Shanghai & - & - & - & - & - & - \\
\hline Shanxi & 0.17 & 3.84 & 3.85 & 26.23 & 0.75 & 21.57 \\
\hline Sichuan & 0.29 & 5.38 & 3.31 & 28.29 & 1.95 & 33 \\
\hline
\end{tabular}




\begin{tabular}{ccccccc} 
Tianjin & - & - & - & - & - & - \\
Xinjiang & 0.06 & 2.97 & 0.24 & 2.68 & 0.12 & 7.83 \\
Yunnan & 0.36 & 8.82 & 1.48 & 42.54 & 0.8 & 73.62 \\
Zhejiang & 0.65 & 12.04 & 12.02 & 17.25 & 0.47 & 24.2 \\
\hline
\end{tabular}

53

54 Table S4 Averaged HMs concentration in consumed coal by province (unit: $\mathrm{g} / \mathrm{t}$ ). ${ }^{2,3,5}$

\begin{tabular}{|c|c|c|c|c|c|c|}
\hline Provinces & $\mathrm{Hg}$ & As & $\mathrm{Se}$ & $\mathrm{Pb}$ & $\mathrm{Cd}$ & $\mathrm{Cr}$ \\
\hline Anhui & 0.4 & 2.94 & 7.19 & 13.82 & 0.16 & 30.37 \\
\hline Beijing & 0.17 & 4.02 & 3.59 & 26.74 & 0.66 & 23.39 \\
\hline Chongqing & 0.32 & 5.74 & 3.7 & 29.91 & 1.19 & 28.44 \\
\hline Fujian & 0.12 & 4.48 & 2.32 & 15.23 & 0.29 & 18.53 \\
\hline Gansu & 0.23 & 3.74 & 0.43 & 7.48 & 0.08 & 21.31 \\
\hline $\begin{array}{c}\text { Guangdon } \\
\mathrm{g}\end{array}$ & 0.14 & 4.04 & 2.78 & 19.2 & 0.53 & 18.67 \\
\hline Guangxi & 0.14 & 6.31 & 1.75 & 14.04 & 0.22 & 44.78 \\
\hline Guizhou & 0.39 & 6.69 & 3.81 & 23.86 & 0.79 & 28.6 \\
\hline Hainan & 0.09 & 2.07 & 2.08 & 14.16 & 0.4 & 11.64 \\
\hline Hebei & 0.19 & 5.17 & 1.94 & 27.45 & 0.25 & 20.71 \\
\hline $\begin{array}{c}\text { Heilongjia } \\
\text { ng }\end{array}$ & 0.16 & 4.39 & 0.97 & 23.76 & 0.12 & 14.97 \\
\hline Henan & 0.19 & 2.64 & 4.58 & 19.47 & 0.59 & 24.61 \\
\hline Hubei & 0.21 & 3.85 & 4.22 & 33.4 & 0.7 & 31.65 \\
\hline Hunan & 0.14 & 8.1 & 3.8 & 25.46 & 0.66 & 31.66 \\
\hline $\begin{array}{c}\text { Inner } \\
\text { Mongolia }\end{array}$ & 0.21 & 5.57 & 1.13 & 25.54 & 0.11 & 12.97 \\
\hline Jiangsu & 0.25 & 4.13 & 3.47 & 26.28 & 0.44 & 24.72 \\
\hline Jiangxi & 0.18 & 5.25 & 6.19 & 22.91 & 0.61 & 33.52 \\
\hline Jilin & 0.28 & 7.77 & 2.38 & 26.86 & 0.15 & 18.1 \\
\hline Liaoning & 0.18 & 5.42 & 1.18 & 21.94 & 0.16 & 20.25 \\
\hline Ningxia & 0.21 & 3.67 & 4.22 & 15.44 & 1.06 & 11.88 \\
\hline Qinghai & 0.24 & 2.89 & 1.16 & 11.44 & 0.26 & 26.46 \\
\hline
\end{tabular}




\begin{tabular}{ccccccc} 
Shaanxi & 0.21 & 3.85 & 3.51 & 33.16 & 0.78 & 30.63 \\
Shandong & 0.19 & 5.05 & 2.76 & 22.77 & 0.37 & 18.26 \\
Shanghai & 0.28 & 4.92 & 2.73 & 24.01 & 0.2 & 17.07 \\
Shanxi & 0.16 & 3.76 & 3.64 & 24.81 & 0.7 & 20.74 \\
Sichuan & 0.29 & 5.45 & 3.27 & 29.64 & 1.74 & 34.39 \\
Tianjin & 0.17 & 3.98 & 3.65 & 26.52 & 0.69 & 22.39 \\
Xinjiang & 0.06 & 3 & 0.25 & 2.93 & 0.12 & 7.88 \\
Yunnan & 0.36 & 8.73 & 1.58 & 41.73 & 0.8 & 71.66 \\
Zhejiang & 0.2 & 4.05 & 3.41 & 27.76 & 0.6 & 24.73 \\
\hline
\end{tabular}

55

56

Table S5 Release ratios of boilers ${ }^{3,5,6}$

\begin{tabular}{ccccccc}
\hline Release ratios (\%) & $\mathrm{Hg}$ & $\mathrm{As}$ & $\mathrm{Se}$ & $\mathrm{Pb}$ & $\mathrm{Cd}$ & $\mathrm{Cr}$ \\
\hline PC & 99.4 & 98.46 & 96.22 & 96.25 & 94.94 & 84.5 \\
CFB & 98.9 & 75.6 & 98.05 & 77.33 & 91.5 & 81.33 \\
\hline
\end{tabular}

57

58

Table S6 Removal efficiency of APCDs for As, $\mathrm{Se}, \mathrm{Pb}, \mathrm{Cd}$ and $\mathrm{Cr}^{3,5}$

\begin{tabular}{cccccc}
\hline Removal efficiency(\%) & $\mathrm{As}$ & $\mathrm{Se}$ & $\mathrm{Pb}$ & $\mathrm{Cd}$ & $\mathrm{Cr}$ \\
\hline ESP & 86.2 & 73.8 & 95 & 95.5 & 95.5 \\
FF & 99 & 65 & 99 & 97.6 & 95.1 \\
WFGD & 80.4 & 74.9 & 78.4 & 80.5 & 86 \\
Coal washing & 54 & 54 & 36.3 & 32.2 & 58 \\
\hline
\end{tabular}

59

60

Table S7 Removal efficiency and speciation profiles of APCDs for $\mathrm{Hg}^{7}$

\begin{tabular}{ccccc}
\hline Removal efficiency(\%) & $\mathrm{Hg}$ & $\mathrm{Hg}^{0}$ & $\mathrm{Hg}^{2+}$ & $\mathrm{Hg}_{\mathrm{p}}$ \\
\hline ESP & 32.4 & $58 \%$ & $41 \%$ & $1.3 \%$ \\
FF & 67.2 & $50 \%$ & $49 \%$ & $0.5 \%$ \\
WFGD+ESP & 60.3 & $84 \%$ & $16 \%$ & $0.6 \%$ \\
WFGD+FF & 86.0 & $78 \%$ & $21 \%$ & $1 \%$ \\
SCR+WFGD+ESP & 70.2 & $72 \%$ & $27 \%$ & $1 \%$
\end{tabular}


Table S8 Distribution characteristics of the APCD removal efficiencies and HMs content ${ }^{2}, 3,5,8$

\begin{tabular}{cccccccc}
\hline \multirow{2}{*}{ Error sources } & Distribution & Parameter & As & Se & Pb & Cd & Cr \\
\hline \multirow{2}{*}{ WFGD } & \multirow{2}{*}{ Triangular } & Maximum & 0.95 & 0.98 & 0.8 & 0.81 & 0.92 \\
& & Minimum & 0.515 & 0.61 & 0.768 & 0.8 & 0.8 \\
& & Mode & 0.8038 & 0.7487 & 0.784 & 0.805 & 0.86 \\
\hline \multirow{2}{*}{ FF } & Maximum & 0.99 & 0.65 & 0.99 & 0.99 & 0.998 \\
& & Minimum & 0.99 & 0.65 & 0.99 & 0.87 & 0.82 \\
\hline \multirow{2}{*}{ ESP } & Noriangular & Average & 0.99 & 0.65 & 0.99 & 0.976 & 0.951 \\
\hline HMs content & Normal & CV & 0.05 & 0.05 & 0.05 & 0.05 & 0.05 \\
\hline
\end{tabular}

63

64 Table S9 HM emissions calculated using HM content in coal consumed

\begin{tabular}{ccccccc}
\hline Region & Hg (t) & As $(\mathbf{t})$ & Se $(\mathbf{t})$ & Pb $(\mathbf{t})$ & $\mathbf{C d}(\mathbf{t})$ & $\mathbf{C r}(\mathbf{t})$ \\
\hline Jiangsu & 1.25 & 4.97 & 8.58 & 11.38 & 0.18 & 8.95 \\
Guizhou & 0.88 & 5.34 & 6.29 & 6.84 & 0.21 & 6.97 \\
Shanxi & 0.57 & 2.63 & 5.42 & 6.32 & 0.17 & 4.52 \\
Sichuan & 0.49 & 3.00 & 3.60 & 5.88 & 0.32 & 5.62 \\
Hebei & 0.59 & 3.65 & 2.77 & 6.96 & 0.06 & 4.38 \\
Shandong & 0.64 & 3.17 & 3.66 & 5.23 & 0.08 & 3.32 \\
Inner Mongolia & 0.67 & 3.79 & 1.56 & 6.26 & 0.02 & 2.58 \\
Liaoning & 0.45 & 2.88 & 1.31 & 4.28 & 0.03 & 3.22 \\
Zhejiang & 0.44 & 1.66 & 2.86 & 4.09 & 0.08 & 2.95 \\
Henan & 0.41 & 1.11 & 4.02 & 2.96 & 0.08 & 3.18 \\
Tianjin & 0.33 & 1.39 & 2.67 & 3.32 & 0.08 & 2.41 \\
Jiangxi & 0.37 & 0.78 & 4.21 & 1.41 & 0.04 & 2.68 \\
Guangdong & 0.34 & 1.63 & 2.32 & 2.78 & 0.07 & 2.29 \\
Yunnan & 0.09 & 1.47 & 0.55 & 2.53 & 0.04 & 3.69
\end{tabular}




\begin{tabular}{ccccccc} 
Chongqing & 0.26 & 1.28 & 1.71 & 2.39 & 0.05 & 1.94 \\
Hubei & 0.21 & 1.08 & 1.79 & 2.50 & 0.05 & 1.98 \\
Shaanxi & 0.28 & 0.85 & 1.64 & 2.64 & 0.05 & 2.02 \\
Jilin & 0.13 & 1.34 & 0.83 & 1.68 & 0.01 & 0.87 \\
Qianghai & 0.15 & 0.72 & 0.96 & 1.35 & 0.03 & 1.09 \\
Heilongjiang & 0.14 & 0.69 & 0.92 & 1.29 & 0.03 & 1.05 \\
Beijing & 0.21 & 0.49 & 1.07 & 1.27 & 0.03 & 0.58 \\
Hunan & 0.09 & 0.43 & 0.76 & 0.87 & 0.02 & 0.87 \\
Xinjiang & 0.07 & 0.97 & 0.16 & 0.34 & 0.01 & 0.71 \\
Anhui & 0.05 & 0.13 & 0.68 & 0.23 & 0.00 & 0.42 \\
Fujian & 0.05 & 0.31 & 0.32 & 0.38 & 0.01 & 0.38 \\
Guangxi & 0.00 & 0.02 & 0.03 & 0.05 & 0.00 & 0.04 \\
Ningxia & 0.00 & 0.02 & 0.03 & 0.04 & 0.00 & 0.04 \\
Gansu & 0.00 & 0.01 & 0.02 & 0.02 & 0.00 & 0.02 \\
\hline
\end{tabular}

65

66 Table S10 HM emissions calculated using HM content in coal locally produced

\begin{tabular}{ccccccc}
\hline Region & Hg (t) & As $(\mathbf{t})$ & Se $(\mathbf{t})$ & Pb $(\mathbf{t})$ & $\mathbf{C d}(\mathbf{t})$ & $\mathbf{C r}(\mathbf{t})$ \\
\hline Jiangsu & 0.21 & 0.49 & 1.07 & 1.27 & 0.03 & 0.58 \\
Guizhou & 0.33 & 1.39 & 2.67 & 3.32 & 0.08 & 2.41 \\
Shanxi & 0.56 & 3.45 & 3.30 & 7.43 & 0.05 & 6.88 \\
Sichuan & 0.64 & 2.69 & 5.73 & 6.68 & 0.18 & 4.70 \\
Hebei & 0.87 & 3.93 & 1.52 & 6.53 & 0.02 & 2.59 \\
Shandong & 0.54 & 2.93 & 0.95 & 3.84 & 0.03 & 4.17 \\
Inner Mongolia & 0.46 & 2.00 & 1.41 & 1.81 & 0.01 & 1.11 \\
Liaoning & 0.25 & 0.75 & 1.21 & 1.24 & 0.02 & 1.19 \\
Zhejiang & 4.52 & 3.30 & 15.11 & 9.09 & 0.02 & 7.17 \\
Henan & 1.61 & 4.92 & 10.10 & 2.54 & 0.06 & 2.88 \\
Tianjin & 0.75 & 3.28 & 4.85 & 3.83 & 0.08 & 3.74 \\
Jiangxi & 0.45 & 0.92 & 4.27 & 2.55 & 0.08 & 3.22 \\
Guangdong & 0.11 & 0.13 & 0.72 & 0.22 & 0.00 & 0.43
\end{tabular}




\begin{tabular}{ccccccc} 
Yunnan & 0.26 & 1.10 & 5.70 & 1.19 & 0.04 & 3.18 \\
Chongqing & 0.22 & 1.11 & 3.71 & 3.54 & 0.02 & 2.54 \\
Hubei & 0.07 & 0.99 & 0.74 & 0.89 & 0.02 & 1.02 \\
Shaanxi & 0.15 & 3.35 & 0.50 & 3.53 & 0.03 & 9.07 \\
Jilin & 0.03 & 0.70 & 0.17 & 0.64 & 0.01 & 0.62 \\
Qianghai & 0.84 & 2.96 & 3.65 & 5.61 & 0.35 & 5.39 \\
Heilongjiang & 0.45 & 1.38 & 2.24 & 2.30 & 0.04 & 2.21 \\
Beijing & 0.01 & 0.03 & 0.04 & 0.04 & 0.00 & 0.04 \\
Hunan & 1.64 & 5.33 & 6.31 & 6.82 & 0.21 & 6.94 \\
Xinjiang & 0.32 & 1.49 & 0.52 & 2.58 & 0.04 & 3.79 \\
Anhui & 0.28 & 0.85 & 1.60 & 2.80 & 0.05 & 2.16 \\
Fujian & 0.00 & 0.01 & 0.02 & 0.02 & 0.00 & 0.02 \\
Guangxi & 0.26 & 0.78 & 1.26 & 1.29 & 0.02 & 1.24 \\
Ningxia & 0.01 & 0.03 & 0.04 & 0.04 & 0.00 & 0.04 \\
Gansu & 0.14 & 0.96 & 0.15 & 0.31 & 0.01 & 0.71 \\
\hline
\end{tabular}

67

68

Table S11 Proportion of unit-specific emission reduction

\begin{tabular}{ccccccc}
\hline Heavy Metal & $\mathrm{Hg}$ & $\mathrm{As}$ & $\mathrm{Se}$ & $\mathrm{Pb}$ & $\mathrm{Cd}$ & $\mathrm{Cr}$ \\
\hline Unit-based emissions & $8.05 \mathrm{t}$ & $39.68 \mathrm{t}$ & $53.31 \mathrm{t}$ & $74.32 \mathrm{t}$ & $1.51 \mathrm{t}$ & $58.89 \mathrm{t}$ \\
\hline Proportion & $87.61 \%$ & $86.56 \%$ & $87.73 \%$ & $86.43 \%$ & $86.81 \%$ & $85.66 \%$ \\
\hline
\end{tabular}

69 Note: The sum of unit-specific HMs reduced is calculated to be $277.71 \mathrm{t}$, accounting for $86.78 \%$ of

70 the total emission reductions by decommissioned CFPPs during 2011-2015.

71 


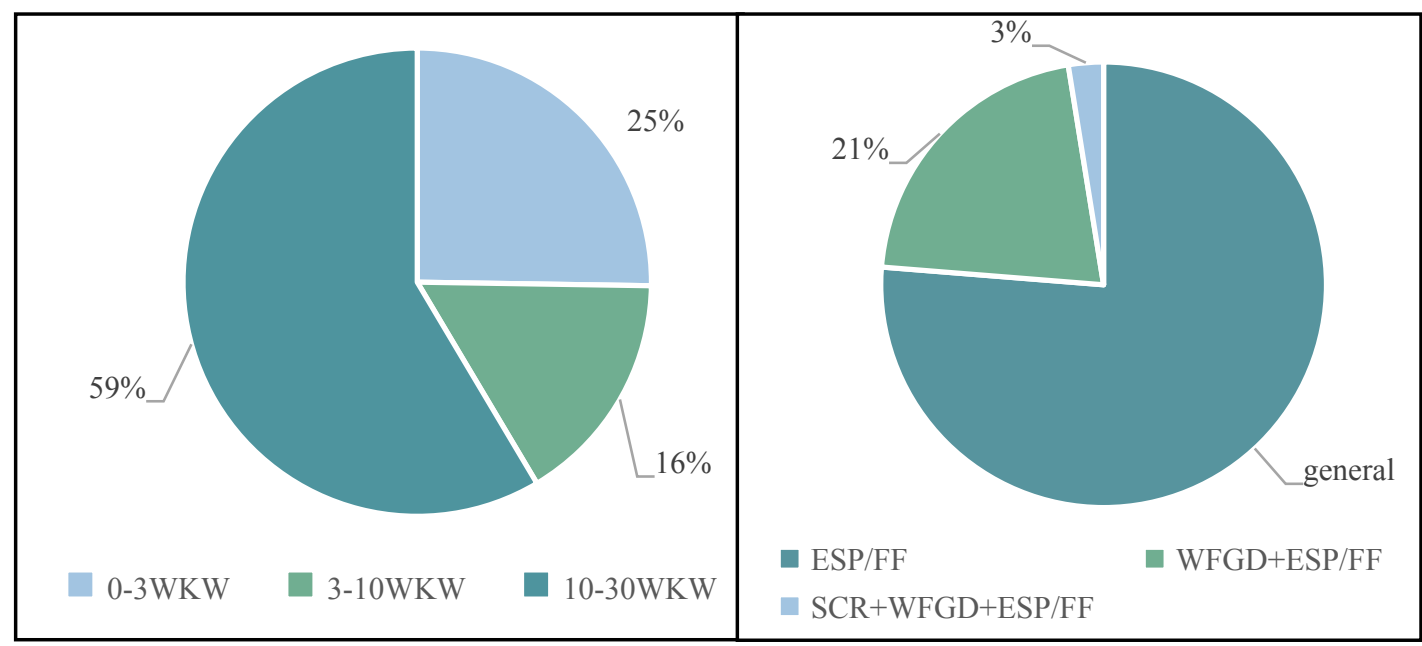

72 73

(a) Capacity range of decommissioned CFPPs

(b) APCD type of decommissioned CFPPs

Figure S1 Parameters of decommissioned CFPPs

74

75

76

77 
79 (1) Meij, R.; te Winkel, H., The emissions of heavy metals and persistent organic pollutants from modern coal-fired power stations. Atmospheric Environment 2007, 41, (40), 9262-9272.

82

(2) Tian, H.; Cheng, K.; Wang, Y.; Zhao, D.; Lu, L.; Jia, W.; Hao, J., Temporal and

83 spatial variation characteristics of atmospheric emissions of $\mathrm{Cd}, \mathrm{Cr}$, and $\mathrm{Pb}$ from coal 84 in China. Atmospheric Environment 2012, 50, 157-163.

85

(3) Tian, H.; Zhu, C.; Gao, J.; Cheng, K.; Hao, J.; Wang, K.; Hua, S.; Wang, Y.;

86 Zhou, J., Quantitative assessment of atmospheric emissions of toxic heavy metals from anthropogenic sources in China: historical trend, spatial distribution,

88 uncertainties, and control policies. Atmospheric Chemistry and Physics 2015, 15, 89 (17), 10127-10147.

90 (4) China Electricity Council The inventory of power plans in China 2010 91 (undocumented report); Beijing, China, 2017 (in Chinese).

92 (5) Tian, H.; Liu, K.; Zhou, J.; Lu, L.; Hao, J.; Qiu, P.; Gao, J.; Zhu, C.; Wang, K.;

93 Hua, S., Atmospheric Emission Inventory of Hazardous Trace Elements from China's 94 Coal-Fired Power Plants-Temporal Trends and Spatial Variation Characteristics.

95 Environmental science \& technology 2014, 48, (6), 3575-3582.

96 (6) Zhu, C.; Tian, H.; Hao, Y.; Gao, J.; Hao, J.; Wang, Y.; Hua, S.; Wang, K.; Liu, H., 97 A high-resolution emission inventory of anthropogenic trace elements in Beijing98 Tianjin-Hebei (BTH) region of China. Atmospheric environment 2018, 191, 452-462.

99 (7) Wu, Q.; Wang, S.; Li, G.; Liang, S.; Lin, C. J.; Wang, Y.; Cai, S.; Liu, K.; Hao, J., 100 Temporal Trend and Spatial Distribution of Speciated Atmospheric Mercury 101 Emissions in China During 1978-2014. Environ Sci Technol 2016, 50, (24), 1342810213435.

103 (8) Zhao, Y.; Wang, S.; Duan, L.; Lei, Y.; Cao, P.; Hao, J., Primary air pollutant 104 emissions of coal-fired power plants in China: Current status and future prediction. 105 Atmospheric Environment 2008, 42, (36), 8442-8452. 